\title{
Surface passivation and boundary lubrication of self-mated tetrahedral amorphous carbon asperities under extreme tribological conditions
}

\author{
Pedro A. ROMERO ${ }^{1}$, Lars PASTEWKA ${ }^{1,2}$, Julian VON LAUTZ ${ }^{1}$, Michael MOSELER ${ }^{1,3,4, *}$ \\ ${ }^{1}$ Fraunhofer Institute for Mechanics of Materials IWM, Wöhlerstraße 11, 79108 Freiburg, Germany \\ ${ }^{2}$ Karlsruhe Institute of Technology, IAM-ZBS, Kaiserstraße 12, 76131 Karlsruhe, Germany \\ ${ }^{3}$ Freiburg Materials Research Center, Stefan-Meier-Straße 21, 79104 Freiburg, Germany \\ ${ }^{4}$ University of Freiburg, Physics Department, Hermann-Herder-Straße 3, 79104 Freiburg, Germany \\ Received: 08 June 2014 / Accepted: 10 June 2014 \\ (C) The author(s) 2014. This article is published with open access at Springerlink.com
}

\begin{abstract}
Tetrahedral amorphous carbon coatings have the potential to significantly reduce friction and wear between sliding components. Here, we provide atomistic insights into the evolution of the sliding interface between naked and hydrogen-passivated ta-C sliding partners under dry and lubricated conditions. Using reactive classical atomistic simulations we show that sliding induces a $\mathrm{sp}^{3}$ to $\mathrm{sp}^{2}$ rehybridization and that the shear resistance is reduced by hydrogen-passivation and hexadecane-lubrication-despite our finding that nanoscale hexadecane layers are not always able to separate and protect ta- $\mathrm{C}$ counter surfaces during sliding. As asperities deform, carbon atoms within the hexadecane lubricant bind to the ta- $\mathrm{C}$ sliding partners resulting in degradation of the hexadecane molecules and in increased material intermixing at the sliding interface. Hydrogen atoms from the passivation layer and from the hexadecane chains continue to be mixed within a sp ${ }^{2}$ rich sliding interface eventually generating a tribo-layer that resembles an a-C:H type of material. Upon separation of the sliding partners, the tribo-couple splits within the newly formed $\mathrm{sp}^{2}$ rich a-C:H mixed layer with significant material transfer across the sliding partners. This leaves behind a-C:H coated ta- $\mathrm{C}$ surfaces with dangling $\mathrm{C}$ bonds, linear $\mathrm{C}$ chains and hydrocarbon fragments.
\end{abstract}

Keywords: Atomic-scale simulations; DLC; lubrication; hexadecane; passivation; sliding; mixed layer; wear

\section{Introduction}

Tetrahedral amorphous carbon (ta-C) exhibits low friction in humid environments and superior resistance to abrasive as well as adhesive wear [1-3]. These tribological properties make ta-C an outstanding candidate for protective coatings that reduce wear and minimize friction in micro- and macromachines [4]. Nevertheless, ta-C coatings abrade slowly, even under lubricated conditions, and the detailed wear mechanisms are typically hidden by complex processes

* Corresponding author: Michael MOSELER.

E-mail: michael.moseler@iwm.fraunhofer.de involving plastic deformation, fracture, changes in surface topography, and loss of surface termination and repassivation at the sliding interface. Experimentally, this is manifested in the formation of a surface layer with a large fraction of $\mathrm{sp}^{2}$-hybridized amorphous carbon $(\mathrm{a}-\mathrm{C})$ atoms that is distinct from the low $\mathrm{sp}^{2}$ but large $\mathrm{sp}^{3}$ (tetrahedral) bulk of the ta-C [5-7].

We here focus on processes that occur at the single asperity scale and will use a simplified model for the tribological contact of two asperities. Figure 1 sketches the anticipated deformation and wear mechanisms during the collision of two such ta- $\mathrm{C}$ asperities under humid environmental conditions as recently proposed by Kunze et al. [8]. As schematically depicted in 


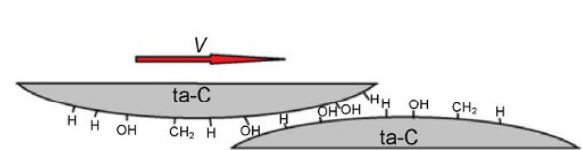

(a) Approach

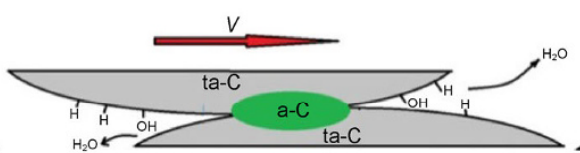

(b) Sliding

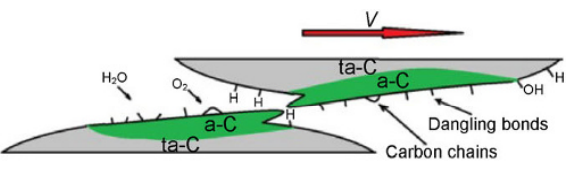

(c) Separation

Fig. 1 Schematic of a ta-C/ta-C asperity contact under humid ambient conditions from (a) the initial asperity collision to (b) the evolution of sliding contact leading to the creation of a mixed a-C:H:O layer until (c) the loss of contact leads to material transfer and the creation of a-C:H:O passivated surfaces with dangling bonds and linear carbon chains (Reproduced with permission from Ref. [8], Copyright Springer, 2013).

Fig. 1(a), ta-C surfaces are passivated with $\mathrm{H}$ and $\mathrm{OH}$ groups after contact with environmental humidity or lubricants [9]. This passivation prevents immediate bonding and cold welding across the counter surfaces.

During sliding at low loads, most of the deformation between the colliding asperities remains within the passivated region around the contact interface. Under these conditions, surfaces are chemically stable and the surface termination remains intact. This usually leads to small frictional resistance [9] in cases with little "overlap" (because of a low applied load) between colliding asperities. Such situations have traditionally been modeled using atomistic techniques by sliding two flat (and therefore compliant) surfaces against each other. These simulations have shown dissociation of individual terminating chemical groups [10], rehybridization of the underlying substrate [11,12], and formation of covalent bonds across interfaces [12, 13].

Generally, contact area and hence overlap (i.e., the fraction of the two asperities that will eventually establish intimate atomic contact) of co-moving asperities increases with increasing load [14-17]. For cases with significant overlapping and interlocking of asperities frictional resistance is typically higher despite the presence of passivation because energy is required for plastic deformation of the subsurface region underneath the initial contact interface.

Such a case is depicted in Fig. 1(b). Rehybridization (conversion of $\mathrm{sp}^{3}$ to $\mathrm{sp}^{2}$ carbon) leads to the formation of a soft amorphous carbon (a-C) layer that accommodates the shear deformation before separation of the asperities. Separation may involve necking and leaves behind two highly reactive (unsaturated) a-C surfaces with dangling bonds and linear $\mathrm{sp}^{1}$ carbon chains $[18,19]$. Reaction of these newly created surfaces with environmental gases can result in chemical wear through the formation of species such as $\mathrm{CO}$ and $\mathrm{CO}_{2}$
$[18,19]$. Recent tribo-experiments with hydrogenated amorphous carbon $(\mathrm{a}-\mathrm{C}: \mathrm{H})$ revealed a similar $\mathrm{sp}^{2}$-rich layer and the creation of chemically active atoms which can react with environmental species [20,21]. Hence, rehybridization and passivation are interrelated processes that strongly influence friction and wear.

We need a clear fundamental understanding of the effects of passivating species, such as hydrogen and oxygen, on the rehybridization mechanism at the sliding interface in order to confirm or reject the scenario depicted in Fig. 1. Atomic-scale simulations can yield such insights since they allow monitoring of the evolution of plastic deformation, rehybridization and repassivation directly. The faithful simulation of ambient humid conditions (i.e., ta-C interacting with $\mathrm{O}_{2}$ or $\mathrm{H}_{2} \mathrm{O}$ ) is currently only possible using quantum chemical methods that are limited to system sizes and time scales too small to provide a full atomistic picture of friction and wear in ta-C $[22,23]$. On the other hand, reliable semi-empirical potentials for hydrocarbon systems [24-26] are readily available for classical atomistic simulations of naked, H-passivated, and lubricated ta-C counter bodies in sliding contact.

In this communication, we apply these classical interatomic potential methods to study the influence of passivation (surface termination) and lubricant on the evolution of the contact interface between ta-C sliding partners. We intentionally prepare atomic-scale models that will experience extreme pressure and shear during sliding, in order to study a situation where severe plastic deformation and intermixing occurs. This is the extreme opposite to wearless sliding of two flat interfaces studied by many authors in the past.

\section{Methods}

We study the evolution of the contact interface between dry and $\mathrm{C}_{16} \mathrm{H}_{34}$ (hexadecane)-lubricated ta-C nano- 
asperities (that are either non-passivated-i.e., nakedor H-passivated) using classical reactive molecular dynamics simulations. While surfaces exposed to the environment are typically terminated, naked surfaces are a model for surfaces dynamically exposed during tribological load after, e.g., the formation of a transfer film that exposes part of the ta-C bulk to the opposing interface. Interatomic interactions were computed using the second generation reactive bond-order (REBO2) potential [24] for hydrocarbon systems. The REBO2 potential used in this work was augmented with screening functions [25-27] in order to properly model the bond-breaking and making processes that typically happen during sliding and plastic deformation.

Small tribo-couples ( $27,000-29,000$ atoms) with a single sinusoidal asperity each were constructed as simplified models for true, typically self-affine and random, surface roughness [28, 29]. The creation of the tribo-couples started with the construction of a ta-C specimen by randomly placing $C$ atoms within a fixed volume to create a random solid of density $3.1 \mathrm{~g} \cdot \mathrm{cm}^{-3}$. This solid was subsequently relaxed with the fast inertial relaxation engine the fast inertial relaxation engine (FIRE) [30] to a force of $10 \mathrm{eV} \cdot \AA^{-1}$ and then heated to $5,000 \mathrm{~K}$ using a Langevin thermostat [31] with a relaxation time constant of $0.015 \mathrm{fs}^{-1}$. After quenching the system to $0 \mathrm{~K}$ within $1.5 \mathrm{ps}$, the ta-C specimens consisted of $60 \% \mathrm{sp}^{3}$ and $40 \% \mathrm{sp}^{2}$ atoms. Here and in the following, the number of $\mathrm{sp}^{3}$ and $\mathrm{sp}^{2}$ atoms was computed by counting the number of 4 -fold and 3-fold coordinated atoms, respectively. Local coordination numbers were computed by counting atoms within a sphere of radius $1.85 \AA$ (the first minimum in the pair distribution function) centered on each atom.

Counter bodies were then created by removing C atoms in a sinusoidal shaped ribbon region in the center of the simulation cell resulting in the creation of two interlocked ta-C asperities. H-passivated surfaces were produced by replacing each $\mathrm{C}-\mathrm{C}$ bond that was cut during the creation of the sinusoidal shape with a $\mathrm{C}-\mathrm{H}$ bond (of appropriately reduced length). In the lubricated cases, hexadecane molecules were placed on a $7 \times 3 \times 2$ grid in $X \times Y \times Z$ directions between the naked and passivated ta-C sliding partners. Here, Z-direction is normal to the surface and sliding happens in the $X$-direction. Interatomic forces within the lubricant and between lubricant and ta-C were computed with the same bond order potential [25] that is used for the interactions within the ta- $C$ counter bodies. Note that because we are operating at high compressive stress, we neglect the influence of nonbonded dispersion interaction that is important at ambient conditions [32].

A thin slab of atoms at the bottom and top of the sliding system was held rigid. The topmost slab of atoms was moved at a constant velocity of $30 \mathrm{~m} \cdot \mathrm{s}^{-1}$ to impose the sliding motion. The remaining atoms were thermostated to $300 \mathrm{~K}$ using a dissipative particle dynamics thermostat $[33,34]$ that is Galilean invariant and therefore does not bias the velocity profile and does not add an artificial drag to the measured friction force.

The tribo-couples were initially relaxed to $300 \mathrm{~K}$ for 5 ps and then compressed by lowering the rigid zone of the upper ta-C counter body until a $\sim 10 \mathrm{GPa}$ normal pressure is established within the mated counter bodies before the onset of sliding. The topmost snapshot of Figs. 2(a), 3(a), 4(a) and 5(a) show the dry/naked, dry/passivated, lubricated/naked and lubricated/passivated tribo-couples, respectively, in their compressed state before sliding. Shearing was started after the normal pressure within the tribocouple stabilized. Once a fully mixed tribolayer formed after approximately 4 ns of sliding, the upper tribo-partner was retracted from the lower one in order to expose the interfacial chemical changes, the sliding interfaces and the material transfer between the ta- $\mathrm{C}$ counter bodies.

\section{Results and discussion}

\subsection{Sliding simulations}

Figures 2, 3, 4 and 5 show key instances during the evolution of sliding between ta-C counter asperities for the four cases considered here (dry/naked, dry/passivated, lubricated/naked and lubricated/ passivated). For every case, the top row of snapshots keeps track of material mixing and transfer by coloring the $\mathrm{C}$ atoms belonging to the upper and lower ta-C counter bodies purple and green, respectively. If present, $\mathrm{H}$-atoms belonging to the surface passivation 

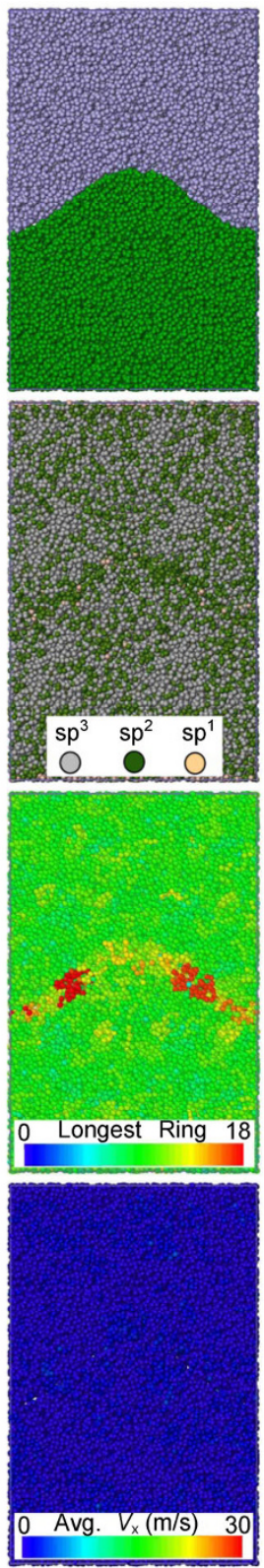

(a) $0.06 \mathrm{~ns}$
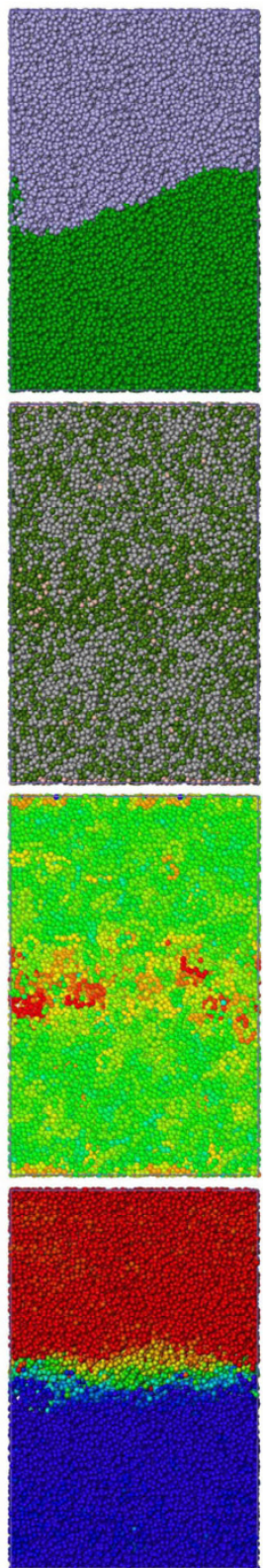

(b) $0.18 \mathrm{~ns}$
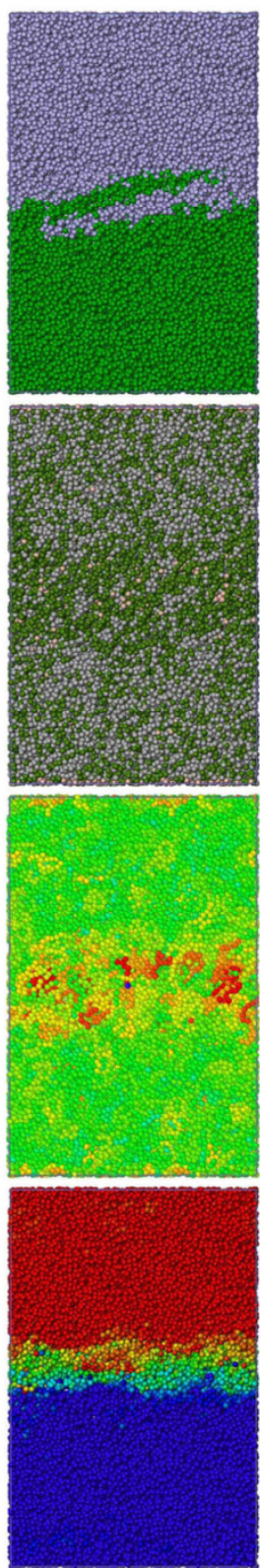

(c) $0.40 \mathrm{~ns}$
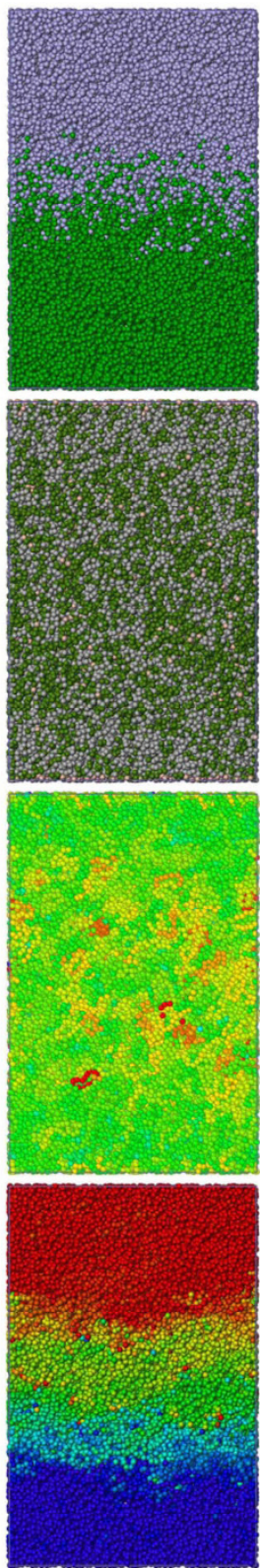

(d) $3.92 \mathrm{~ns}$
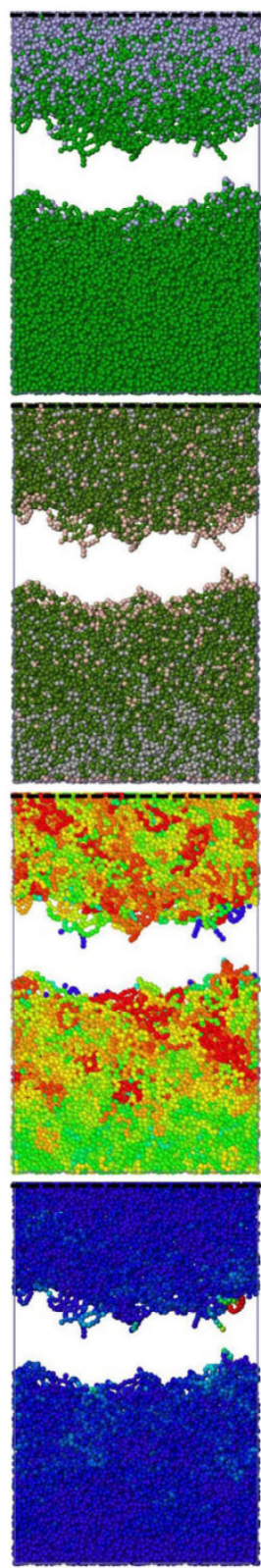

(e) $4.07 \mathrm{~ns}$

Fig. 2 The progression of dry sliding between ta-C counter asperities. Column (a) shows that cold welding already occurs during the pressing stage. Column (b) shows that sliding leads to plowing and tilted surface formation. Column (c) shows the onset of material mixing by the generation of two elongated flakes that become embedded in the opposite counter surfaces. Column (d) presents the fully developed atomically mixed layer at the sliding interface exhibiting significant $\mathrm{sp}^{3}$ to $\mathrm{sp}^{2}$ rehybridization. Column (e) presents the separation of the counter bodies leading to rupture along the $\mathrm{sp}^{2}$ rich layer and the formation of a-C covered surfaces with dangling bonds and linear carbon chains.

are colored yellow and $\mathrm{C} / \mathrm{H}$ atoms from the lubricant molecules are colored red/light-blue. The degree of rehybridization is presented in the second row of snapshots, where $\mathrm{C}$ atoms have been colored according to their hybridization state with grey/green/ivory denoting $\mathrm{sp}^{3} / \mathrm{sp}^{2} / \mathrm{sp}^{1} \mathrm{C}$ atoms, respectively. Note that hybridization is a measure that is accessible in experiments, e.g., through Raman spectroscopy [35].

In addition to the hybridization we compute the statistics of local "shortest-path" rings [36] as a measure for intermediate-range correlation. Each atom can be a member of multiple rings, and we color each atom 

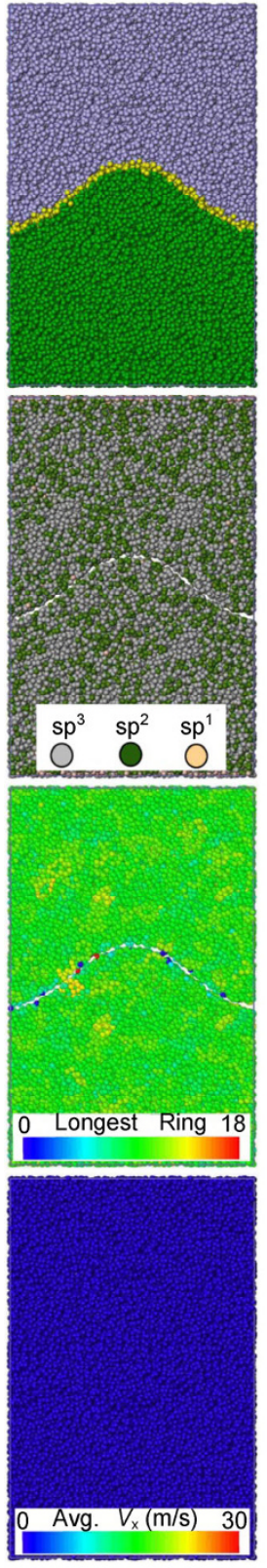

(a) $0.06 \mathrm{~ns}$
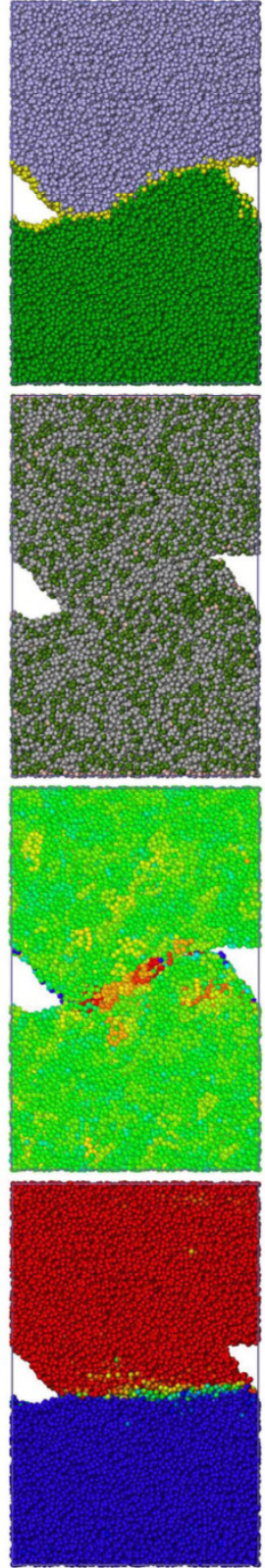

(b) $0.17 \mathrm{~ns}$
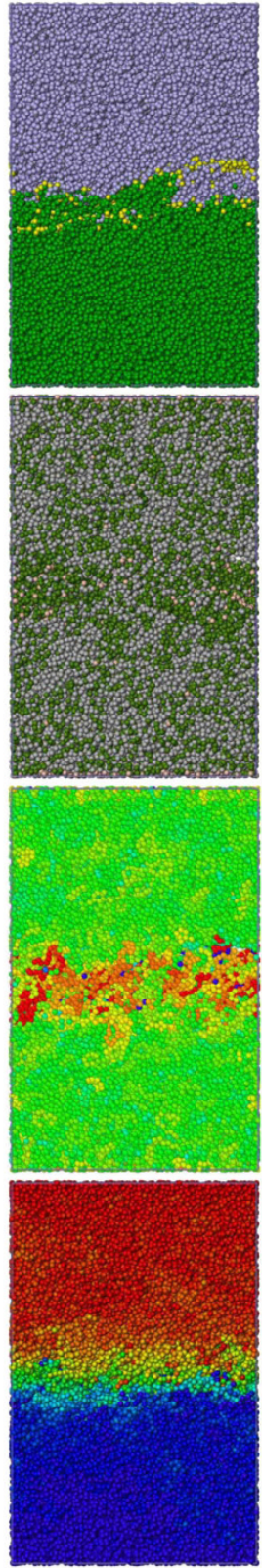

(c) $0.38 \mathrm{~ns}$
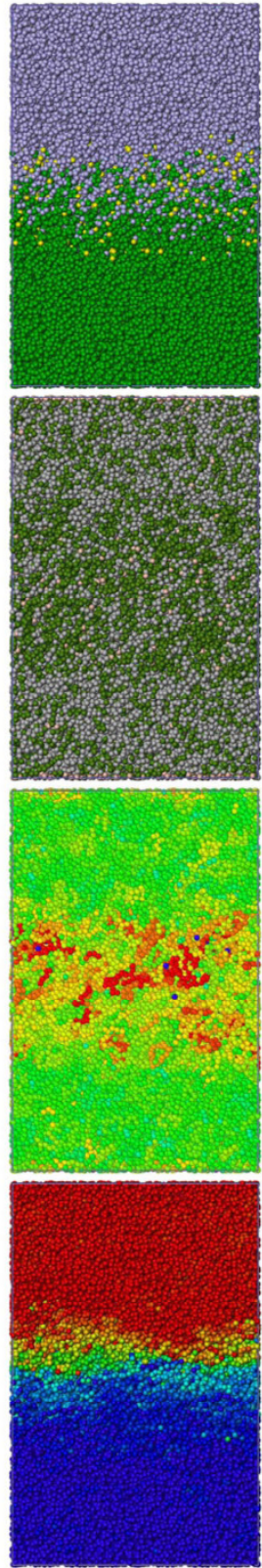

(d) $3.98 \mathrm{~ns}$
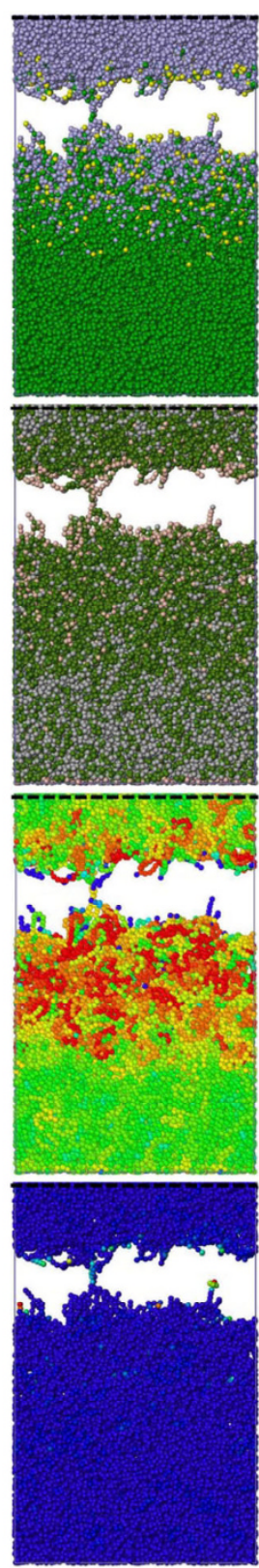

(e) $4.08 \mathrm{~ns}$

Fig. 3 The progression of sliding between H-passivated (yellow colored atoms) ta-C counter asperities. Column (a) shows that hydrogen passivation prevents cold welding during the pressing stage. Column (b) shows that the onset of sliding leads to surface plowing and tilted surface formation. Column (c) shows the generation of hydrogen wrapped elongated wear debris. Column (d) presents the developed sliding interface consisting of a $\mathrm{sp}^{2}$ rich a-C:H like tribolayer. Column (e) presents the separation of the counter bodies, which leads to rupture between the $\mathrm{sp}^{2}$ rich layer and the formation of a-C:H passivated surfaces with dangling bonds and linear carbon chains.

according to the length of the longest C-ring that it forms (third row of snapshots). As will be seen below, this local atomic measure is a sensitive indicator for the structural changes imposed by the shearing motion that generally lead to an increase in ring sizes. Additionally, lubricant molecules are linear and do not participate in any ring, giving zero for the length of the longest C-ring that lubricant carbon atoms form. Since during sliding lubricant molecules start to degrade and form rings, this measure can be used to identify the remaining intact lubricant molecules.

Lastly, as a means of locating the sliding interface, 

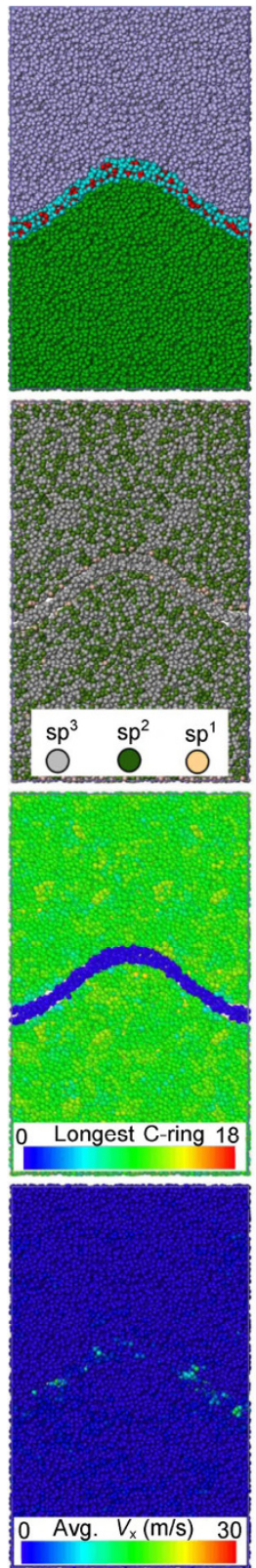

(a) $0.06 \mathrm{~ns}$
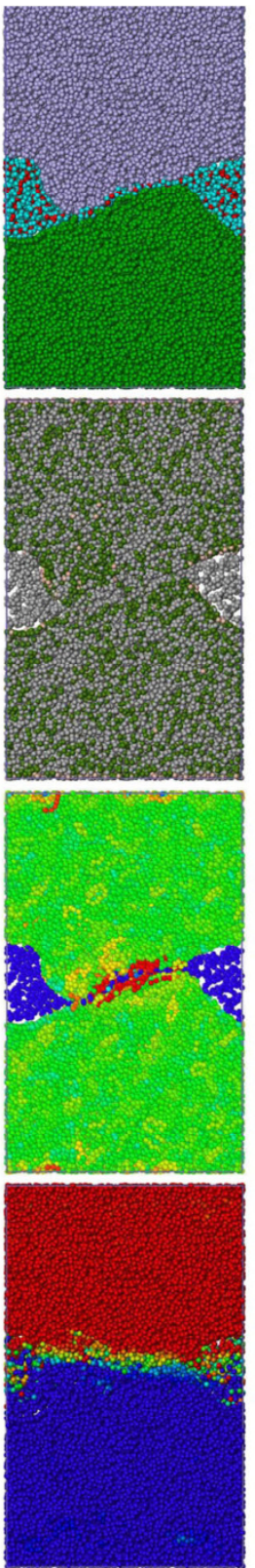

(b) $0.18 \mathrm{~ns}$
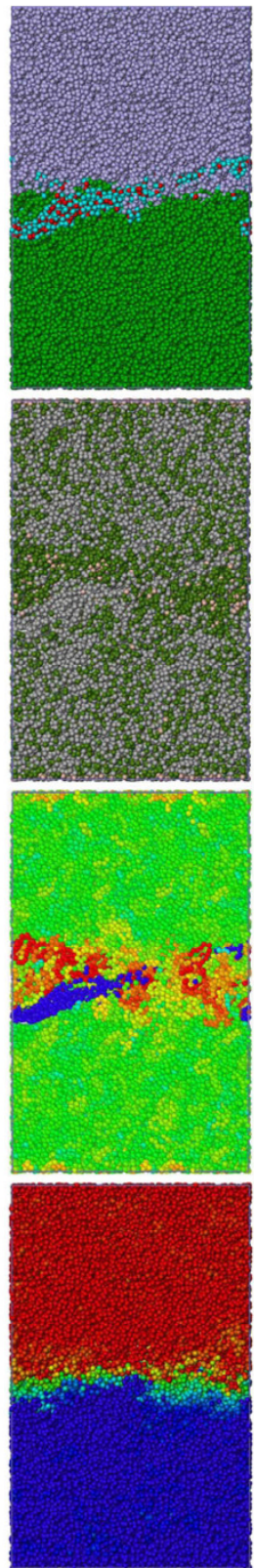

(c) $0.40 \mathrm{~ns}$
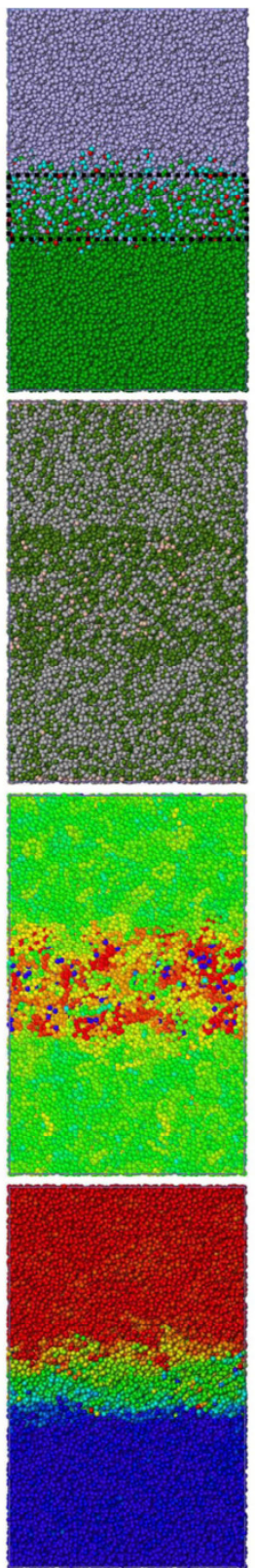

(d) $3.93 \mathrm{~ns}$
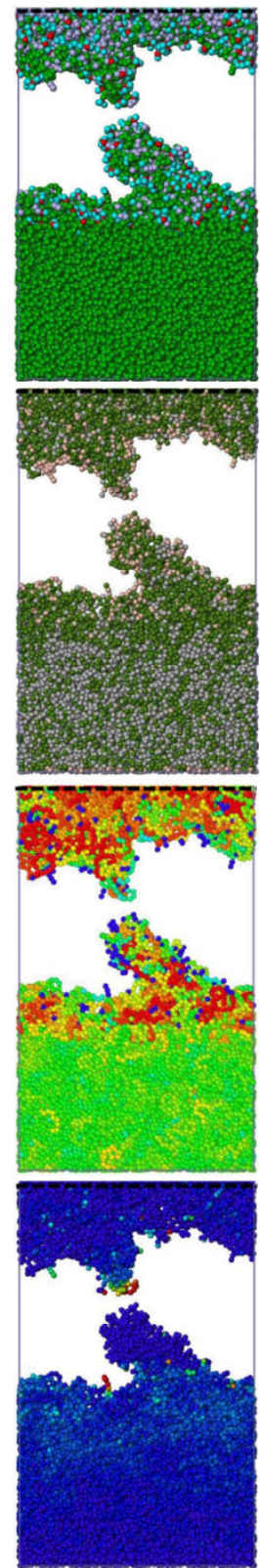

(e) $4.08 \mathrm{~ns}$

Fig. 4 The progression of hexadecane (red/light-blue colored $\mathrm{C} / \mathrm{H}$ atoms in $\mathrm{C}_{16} \mathrm{H}_{34}$ molecules) lubricated sliding between ta-C counter asperities. Column (a) shows that hexadecane lubrication prevents bonding and cold welding across the counter surfaces during the compression stage. Column (b) shows the onset of sliding leading to lubricant squeezing, plowing and inclined surface formation. Column (c) shows the generation of wear debris wrapped with lubricant. Column (d) presents the developed $\mathrm{sp}^{2}$ rich a-C:H tribo- layer. Column (e) presents the separation of the counter bodies leading to rupture within the $\mathrm{sp}^{2}$ rich layer and the formation of a-C:H coated surfaces with dangling bonds and a-C:H debris columns.

the fourth row of snapshots depicts the atoms according to their local average horizontal velocity which is calculated from the atomic change in displacement after every 5 ps. For every system column (a) (Figs. 2(a)-5(a)) presents the relaxed and pressed state at the end of the compression stage. Column (b)
(Figs. 2(b)-5(b)) shows that the onset of sliding leads to severe plastic deformation and initial flattening of the asperities for all tribo-couples. Column (c) (Figs. 2(c)-5(c)) shows an instant where wear debris are first generated. Column (d) (Figs. 2(d)-5(d)) presents the final intermixed sliding interface after about $4 \mathrm{~ns}$ 

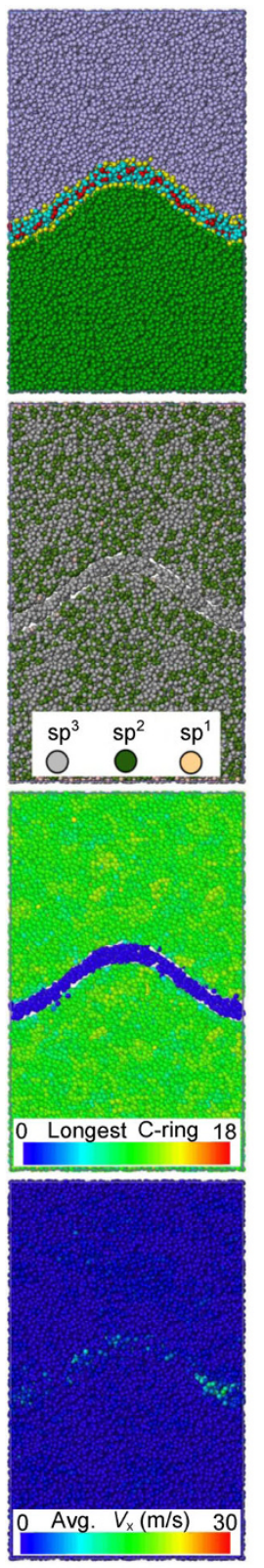

(a) $0.06 \mathrm{~ns}$
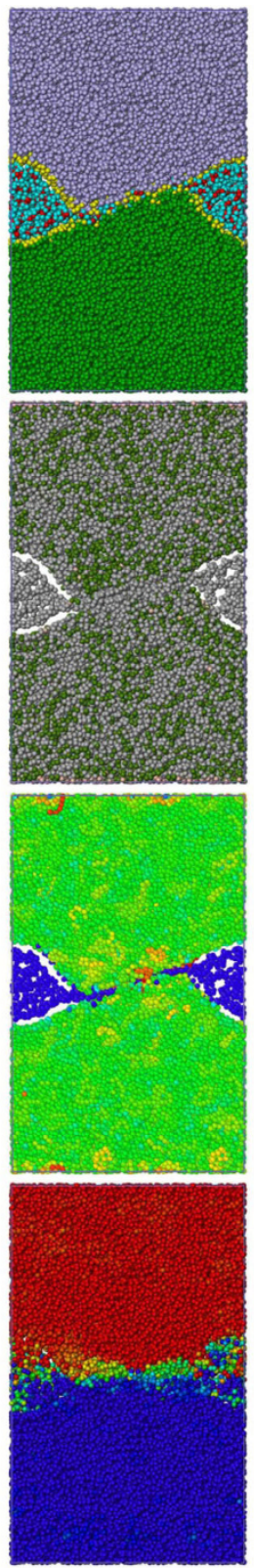

(b) $0.18 \mathrm{~ns}$
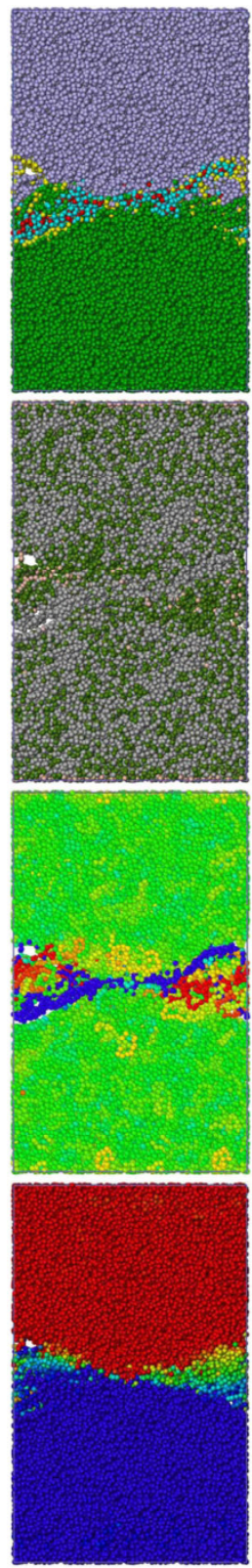

(c) $0.39 \mathrm{~ns}$
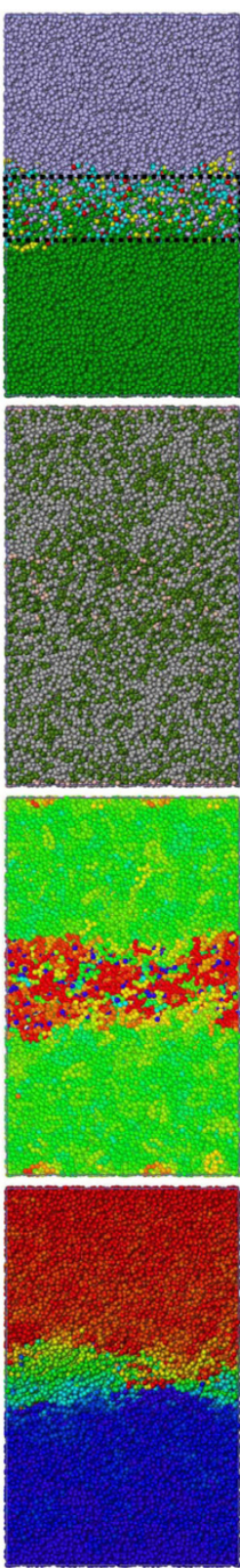

(d) $3.94 \mathrm{~ns}$
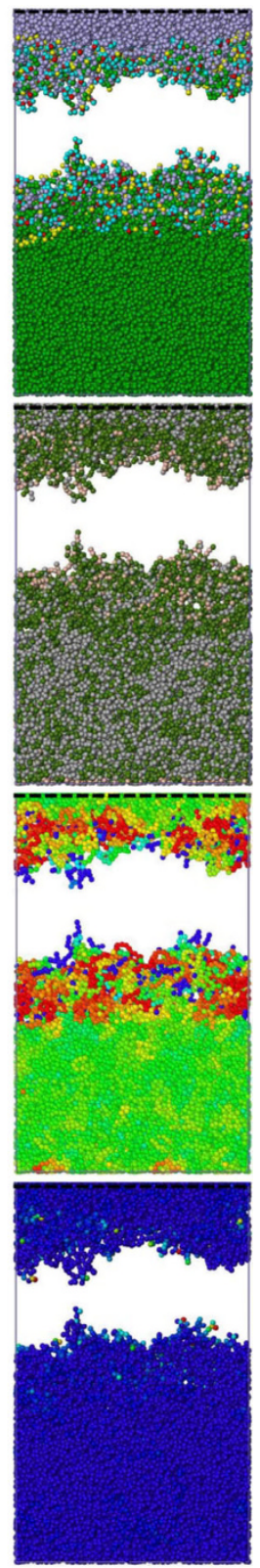

(e) $4.06 \mathrm{~ns}$

Fig. 5 The progression of hexadecane (red/light-blue colored $\mathrm{C} / \mathrm{H}$ atoms in $\mathrm{C}_{16} \mathrm{H}_{34}$ molecules) lubricated sliding between H-passivated (yellow colored atoms) ta-C counter asperities. Column (a) shows that $\mathrm{H}$-passivation along with $\mathrm{C}_{16} \mathrm{H}_{34}$-lubrication prevent bonding and cold welding across the counter surfaces during the pressing stage. Column (b) shows the onset of sliding leading to lubricant squeezing, plowing and inclined surface formation. Column (c) shows the generation of wear debris wrapped in $\mathrm{H}_{\text {and }} \mathrm{C}_{16} \mathrm{H}_{34}$. Column (d) presents the developed sliding interface exhibiting a sp ${ }^{2}$ rich a-C:H tribo-layer. Column (e) presents the separation of the counter bodies leading to rupture along the $\mathrm{sp}^{2}$ rich layer and the formation of a-C:H coated surfaces with dangling bonds and small carbon chains.

of sliding. Lastly, column (e) (Figs. 2(e)-5(e))presents the separation of the counter bodies, which leads to rupture within the developed tribo-layer. Note that the top part of the upper counter body is not shown in Figs. 2(e)-5(e).

\subsubsection{Dry sliding, naked surfaces}

Sliding contact between two naked ta-C asperities under dry vacuum sliding conditions is presented in Figure 2. As shown in Fig. 2(a), the asperities cold weld at initial contact through $\mathrm{C}-\mathrm{C}$ bonds that form 
across the interface. The longest carbon rings in the system can be found where the counter bodies are first brought into interlocked contact by compression. At the onset of sliding, the cold-welded asperities deform plastically close to the initial contact interface (see Fig. 2(b)). The top panel of Fig. 2(b) shows that this initial sliding deformation continuously deforms the asperities to form an almost straight-but tilted with respect to the sliding plane-interface between the two counter bodies. During this stage, the concentration of non-sp ${ }^{3}$ hybridized atoms and the length of C-rings near the sliding interface increase considerably. The initial contact line is then deformed severely into flakelike structures (see top panel of Fig. 2(c)) which must be due to a mixing motion. Finally, the contact line, that was initially atomically sharp, becomes diffused as the atoms from both asperities intermix and spreads out over a width a couple of atomic diameters during sliding (see top panel of Fig. 2(d)). The progression of the intermixing shown in the top row of Fig. 2 is reminiscent of calculations on the sliding of model metallic glasses [37, 38].

As shown in Fig. 2(d), a thick sp ${ }^{2}$ rich a-C layer with a high concentration of long C-rings is eventually established at the sliding interface from $\mathrm{C}$ atoms belonging to both counter bodies. The local velocity profile of Fig. 2(d) indicates, that this $\mathrm{sp}^{2}$ rich region $[12,39-41]$ serves as a tribo-layer where the sliding motion is accommodated.

Finally, as shown in Fig. 2(e), separation of the sliding partners occurs somewhere within this damaged shear accommodation zone. It generally leads to material exchange between the counter bodies and may lead to a net transfer to one of the surfaces. The exposed, newly created surfaces have a surface structure that consists of dangling bonds and linear carbon chains $[18,19]$. We note that the full separation of the surfaces was preceded by void formation and necking that is common in the failure of ductile amorphous materials $[42,43]$. The panels in Fig. 2(e) also show that this fracture at the sliding interface further increases the $\mathrm{sp}^{2}$ content and the ring length in an extended region around the initial sliding interface.

\subsubsection{Dry sliding, passivated surfaces}

Figure 3 presents the case of sliding contact between two ta-C asperities where surface dangling bonds have been passivated with hydrogen. Unlike the case of naked surfaces presented in Fig. 2, the asperities do not cold weld at the initial contact interface because the hydrogen termination separates the surfaces when the counter bodies are first brought into interlocked contact by the external load. The lack of coldwelding across the initial contact interface is evident during the onset of sliding where separation of the two surfaces is clearly visible (see Fig. 3(b)). Plastic deformation then leads to the formation of a straight but tilted interface between the counter bodies (top panel of Fig. 3(b)) resembling those proposed in Fig. 1(c) and similar to the naked case. The passivated case shows a noticeable increase in the concentration of non-sp $\mathrm{p}^{3}$ hybridized atoms and the length of C-rings only at the contacting asperity peaks, and not along the full sliding interface during the onset of sliding (Fig. 3(b)). Towards the end of the initial deformation of the asperities, shear is localized below the surface (i.e., within the asperity itself) as can be seen from the local velocity profile in the bottom panel of Fig. 3(b). During plastic deformation, potential wear debris form by wrapping worn ta-C material with hydrogen atoms while the concentration of non-sp ${ }^{3}$ hybridized atoms and the C-ring size increase around the sliding interface (see Fig. 3(c)). The resulting debris with reduced $\mathrm{sp}^{3}$ continue to be smeared within the sliding interface until a mixed a-C: $\mathrm{H}$ layer with a high concentration of non-sp ${ }^{3}$ atoms and long C-rings forms (see Fig. 3(d)).

The velocity profiles in Figs. 2(d) and 3(d) indicate that passivation reduces the thickness of the dynamically formed and $\mathrm{sp}^{2}$ rich tribo-layer. Eventual separation of the sliding partners leads to material transfer between the counter bodies and to the formation of dangling bonds and long linear carbon chains on the newly created surfaces $[18,19]$ (see Fig. 3(e)). Void formation and necking is in this case less severe than for the naked surfaces presented in Fig. 2. The panels in Fig. 3(e) also show that upon separation of the contact, failure occurs within the $\mathrm{sp}^{2}$ rich a-C:H mixed layer. Surface creation is accompanied by an increase in volume that leads to an increase in the concentration of long C-rings and to an increase in $\mathrm{sp}^{2}$ and $\mathrm{sp}^{1}$ carbon atoms. 


\subsubsection{Lubricated sliding, naked surfaces}

Figure 4 presents the progression of sliding between naked ta-C counter asperities under hexadecane boundary lubrication. Close inspection of Fig. 4(a) reveals that the hexadecane molecules maintain their linear structure as contact is first established. Hexadecane is therefore able to prevent bonding and hence cold welding across the counter ta-C surfaces. However, during the onset of sliding most of the hexadecane molecules are squeezed into the valleys leading to a depletion of hexadecane at the contact spots that feel the highest pressure. During this period, the sliding interface is located within the squeezed lubricant and the deforming ta-C asperity peaks (see the local velocity profile in the bottom panel of Fig. 4(b)) The asperities eventually form wear debris that are partially wrapped by hexadecane molecules (see Fig. 4(c)).

An $\mathrm{sp}^{2}$ rich a-C:H tribo layer forms as the hexadecane molecules continue to decompose and mix with the worn ta-C material (see Fig. 4(d)). The sliding interface is located in the region where the longest $\mathrm{C}$-rings are found (see Fig. 4(d)). Similar to both dry cases, final retraction of the ta-C counter bodies leads to the formation of free surfaces within the $\mathrm{sp}^{2}$ rich a-C:H tribolayer (see Fig. 4(e)). Full surface separation is preceded by void formation and necking due to binding of hexadecane molecules to the opposing ta-C surfaces during retraction. Finally, as can be seen in the top panel of Fig. 4(e), separation of the counter bodies leads to the formation of a-C:H terminated ta- $\mathrm{C}$ surfaces with dangling $C$ bonds and partial hydrogen passivation.

\subsubsection{Lubricated sliding, passivated surfaces}

In the fourth case, both ta-C counter surfaces have been passivated and hexadecane is introduced between the surfaces (Fig. 5). Hydrogen surface termination along with the hexadecane molecules prevent the counter ta-C surfaces from cold welding during the compression stage (see Fig. 5(a)). This is the same behavior already observed for passivated surfaces and dry sliding, or for bare surfaces in the presence of hexadecane molecules. The onset of sliding leads to the plastic deformation of the asperities and an almost flat but tilted interface between the surfaces. However, even though most of the hexadecane mole- cules are squeezed out of the contact region into the valleys, the counter surfaces remain unbonded due to passivation.

During the initiation of sliding, no significant formation of $\mathrm{C}$-rings involving hexadecane $\mathrm{C}$ atoms or $\mathrm{C}$ atoms across the counter surfaces is observed (see Fig. 5(b)). This is unlike the lubricated case with naked surfaces, where surfaces immediately cold weld to form larger rings. The formation of C-rings that involve carbon from the hexadecane lubricant happens once the asperity peaks are worn off to form debris flakes wrapped by $\mathrm{H}$ and hexadecane molecules that are subsequently smeared along the sliding interface (see Fig. 5(c)).

Similar to the lubricated/naked case, during the initial stages, the sliding interface is heavily localized within the layer of hexadecane molecules. This is evident from the local velocity profiles in Figs. 5(b) and 5(c). Less localization is observed for both dry cases. The progression of sliding generates an $\mathrm{sp}^{2}$ rich a-C:H layer with a large portion of the original hexadecane molecular chains transformed into C-rings. Notice that as in the previous cases, the longest C-rings are localized within the mixed a-C:H layer that accommodates the sliding motion. As before, retraction of the counter bodies leads to the generation of two a-C:H covered ta- $\mathrm{C}$ counter bodies.

\subsection{Normal pressure, shear stress and rehybridiza- tion}

Figure 6 compares the evolution (from compression to sliding) of the normal pressure, shear stress and degree of rehybridization for the four different tribocouples presented in Figures 2, 3, 4 and 5. As shown in Fig. 6(a), all four cases relax to around $10 \mathrm{GPa}$ pressure within the first 55 ps. The pressure quickly increases at the onset of sliding as the asperity peaks are forced to traverse over each other and deform. This leads to maximum material compression (see for instance Figs. 2(b), 3(b), 4(b) and 5(b)) and high pressure.

At about $200 \mathrm{ps}$ both the dry/naked and dry/ passivated cases reach an initial maximum normal pressure of $\sim 50 \mathrm{GPa}$ while both hexadecane lubricated cases (i.e., lubricated/naked and lubricated/passivated) reach a noticeably lower maximum of $\sim 40 \mathrm{GPa}$. Except for the dry/naked case where both tribopartners cold 


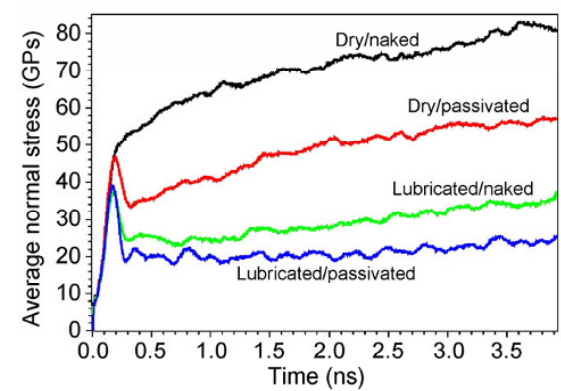

(a) Normal pressure

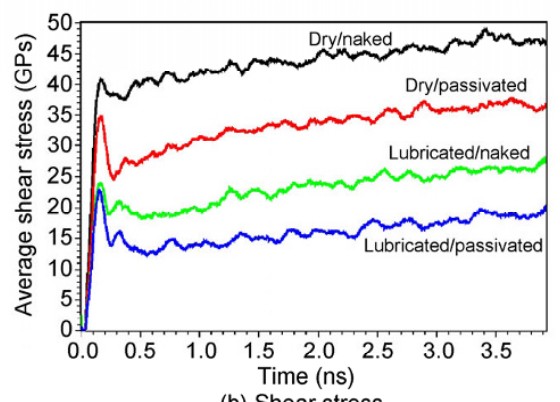

(b) Shear stress

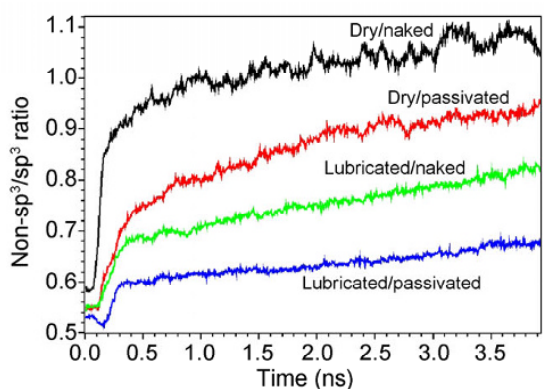

(c) Non-sp $\mathrm{s}^{3}$ to $\mathrm{sp}^{3}$ ratio

Fig. 6 Evolution of the (a) normal pressure, (b) shear stress and (c) ratio of non- $\mathrm{sp}^{3}$ to $\mathrm{sp}^{3}$ atoms during the pressing and sliding stages of dry/naked, dry/passivated, lubricated/naked, and lubricated/passivated ta-C nano-asperity contacts.

weld right from the beginning, all cases undergo a drop in the normal pressure after this maximum when the asperity peaks have slid past each other for the first time. Both lubricated cases then settle at normal pressures around $20 \mathrm{GPa}$ after this drop with only a small progressive increase after $\sim 2.0 \mathrm{~ns}$. The dry cases never settle and continuously show a progressive increase in pressure after the initial drop. This is due to the fact that $\mathrm{sp}^{2}$ atoms require a larger equilibrium volume than $\mathrm{sp}^{3}$ atoms. During $\mathrm{sp}^{3}$ to $\mathrm{sp}^{2}$ rehybridization the system tries to expand its local volume. Since the total volume is confined due to the constant height sliding conditions, the total pressure increases. On the other hand, the lubricated cases form an a-C:H layer, with slower $\mathrm{sp}^{3}$ to $\mathrm{sp}^{2}$ rehybridization and consequently less volume expansion.

Our simulations were intentionally set up to the largest degree of interlocking of the two asperities to drive the system into the most severe situation experienced in a tribo-contact. The simulations therefore show high shear resistance to the imposed sliding motion (Fig. 6(b)). At the onset of sliding, the shear responses of the dry tribo-couples increase more rapidly than the lubricated ones. While the lubricated cases reach a maximum shear stress of $\sim 24 \mathrm{GPa}$, the dry/passivated case reaches a value of $\sim 36 \mathrm{GPa}$ and the dry/naked case reaches an even higher value of $\sim 42 \mathrm{GPa}$. All cases, with the exception of the dry/naked one, experience a significant drop in shear stress once the asperities have been plastically deformed and flattened. After flattening of the asperities, both dry cases exhibit a slight but continuous increase in shear stress as sliding under constant height continues and the normal pressure increases due to the $\mathrm{sp}^{3}$ to $\mathrm{sp}^{2}$ transformation. On the other hand, the lubricated cases appear to first settle at shear stresses of $\sim 19$ GPa and $\sim 13 \mathrm{GPa}$, respectively with only minor progressive increases after $\sim 1.3 \mathrm{~ns}$. This is due to the formation of a layer composed of hexadecane molecules and passivated a-C clusters between the counter surfaces. This tribo-layer accommodates shear, and has a lower shear resistance than bulk ta-C or the soft a-C tribo layer that forms in the dry/naked simulations.

Figure 6(c) shows the $\mathrm{sp}^{3}$ to $\mathrm{sp}^{2}$ rehybridization of carbon bonds during tribological sliding. During the pressing stage, the dry/naked tribo-couple exhibits the highest ratio of non-sp ${ }^{3}$ to $\mathrm{sp}^{3} \mathrm{C}$ atoms while the lubricated/passivated case shows the lowest starting ratio of non-sp $\mathrm{p}^{3}$ to $\mathrm{sp}^{3} \mathrm{C}$ atoms. In the dry/naked case, cold welding at the initial contact interface leads to an increase in $\mathrm{sp}^{3} \mathrm{C}$ atoms but in the lubricated/ passivated case cold welding is prevented during the pressing stage by the hydrogen passivation and hexadecane lubrication. The degree of hybridization across the initial contact interface during pressing can see in Figs. 2(a), 3(a), 4(a), and 5(a). The dry/naked case undergoes $\mathrm{sp}^{3}$ to $\mathrm{sp}^{2}$ rehybridization at a higher rate than the dry/passivated and lubricated systems during the initial stage of sliding from 55 ps to around $250 \mathrm{ps}$. The lubricated/passivated system even experiences an increases in $\mathrm{sp}^{3} \mathrm{C}$ atoms from $\sim 55 \mathrm{ps}$ to $\sim 150$ ps due to compression of the asperity peaks prior to flattening. After their initial rapid increase up to $200 \mathrm{ps}$, both dry cases continue to increase their non-sp $\mathrm{p}^{3}$ to $\mathrm{sp}^{3}$ ratio although at a lower rate. For the lubricated cases, the non-sp $\mathrm{p}^{3}$ to $\mathrm{sp}^{3}$ ratio almost remains constant from $\sim 400$ ps to 1200 ps with only a minor overall increases after $\sim 1200$ ns relative to the dry cases. 


\section{3 a-C:H tribolyer}

We hypothesize that both hexadecane lubricated systems eventually establish a mixed a-C:H like layer which accommodates the sliding shear. This in turn greatly reduces the degree of $\mathrm{sp}^{3}$ to $\mathrm{sp}^{2}$ rehybridization along with the shear resistance. In essence, a lubricated ta-C/ta-C tribocouple appears to slide in a manner similar to that of a dry a-C:H/a-C:H tribo-couple. We now analyze these mixed a-C:H layers (which have been marked with a dashed rectangle in the top panel of Figs. 4(d) and 5(d)] in more detail. Figures 7 and 8 present close-up views of the a-C:H layer generated by both lubricated cases. Figures 7(a) and 8(a) show that both lubricated systems establish a-C:H mixed layers with 4.3:1 and 2.5:1 carbon to hydrogen ratios for the lubricated/naked and the lubricated/passivated surfaces, respectively. Note, that $\mathrm{H}$ atoms are well

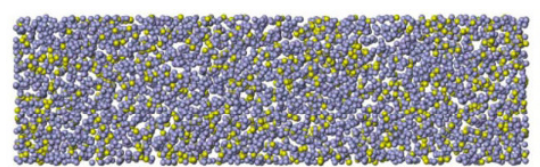

(a) $\sim 4.3: 1 \mathrm{C}: \mathrm{H}$ ratio, a-C:H layer

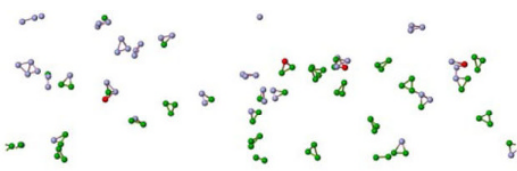

(d) 3-member rings

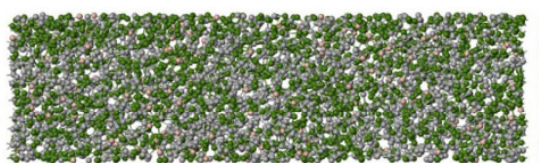

(b) $~ 5: 4$ non- $\mathrm{sp}^{3}: \mathrm{sp}^{3}$ ratio

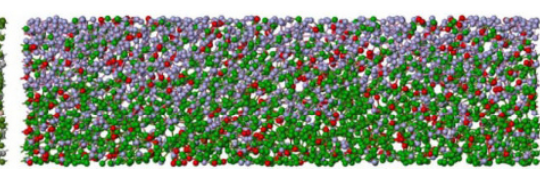

(c) $\mathrm{C}$ atom mixing, $\mathrm{C}-\mathrm{C}$ bonds

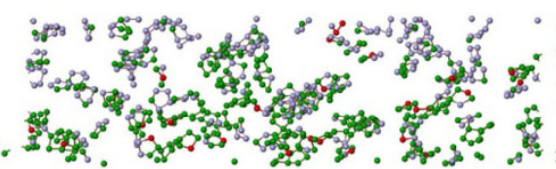

(e) 5-member rings

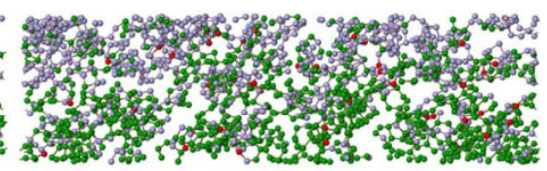

(f) 7-member rings

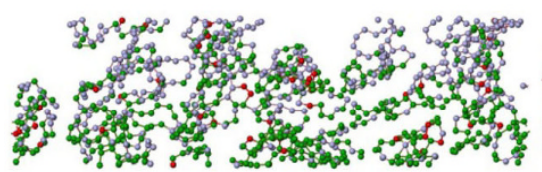

(g) 15-member rings

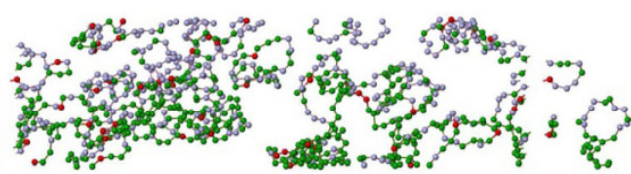

(h) 17-member rings

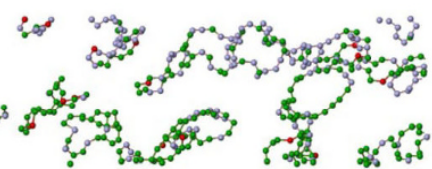

(i) 19-member rings

Fig. 7 The developed mixed a-C:H layer within the lubricated/naked tribo couple after $\sim 4$ ns. (a) Mixed a-C:H layer with a $\sim 4.3: 1$ carbon to hydrogen ratio where all carbon atoms are purple colored and every hydrogen atom is yellow colored. (b) The hybridization of the carbon atoms within the mixed layer showing a $\sim 5: 4$ non- $\mathrm{sp}^{3}$ to $\mathrm{sp}^{3}$ ratio. (c) The mixing of carbon atoms from the green bottom asperity, the purple top asperity and the red hexadecane C atoms. (d)-(i) Sample carbon rings within the layer shown in (d). Only the carbon atoms are displayed in (b) to (i).

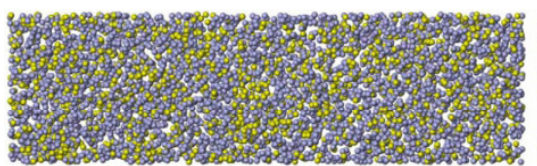

(a) 2.5:1 C:H ratio, a-C:H layer

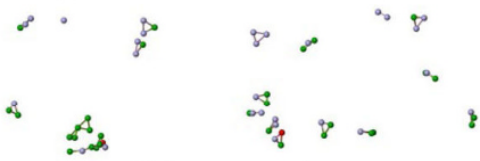

(d) 3-member rings

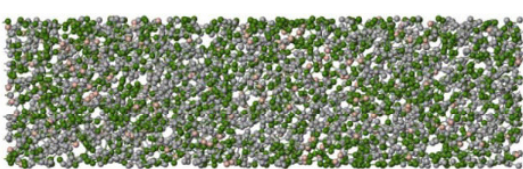

(b) 1:1 non-sp $\mathbf{s p}^{3} \mathrm{sp}^{3}$ ratio

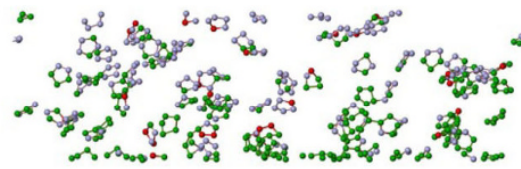

(e) 5 -member rings

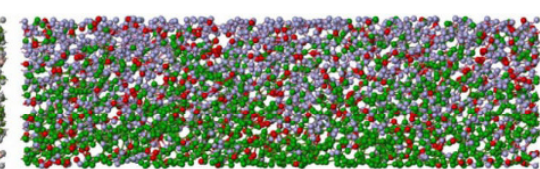

(c) $\mathrm{C}$ atom mixing, $\mathrm{C}-\mathrm{C}$ bonds

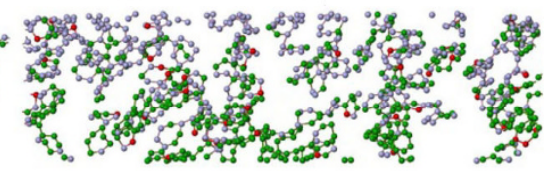

(f) 7-member rings

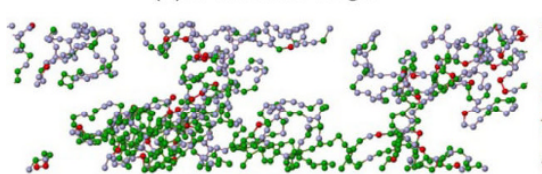

(g) 15-member rings

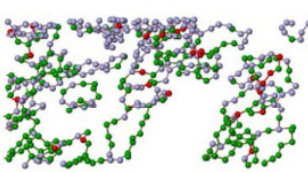

(h) 17-member rings

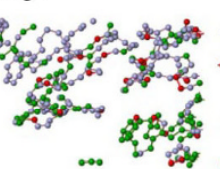

(1)

Fig. 8 The developed mixed a-C:H layer within the lubricated/passivated tribo couple after $\sim 4$ ns. (a) Mixed a-C:H layer with a $\sim 2.5: 1$ carbon to hydrogen ratio where all carbon atoms are purple colored and every hydrogen atom is yellow colored. (b) The hybridization of the carbon atoms within the mixed layer showing a sim 1:1 non-sp ${ }^{3}$ to $\mathrm{sp}^{3}$ ratio. (c) The mixing of carbon atoms from the green bottom asperity, the purple top asperity and the red hexadecane C atoms. (d)-(i) Sample carbon rings within the layer shown in (d). Only the carbon atoms are displayed in (b) to (i). 
spread and mixed within the a-C atoms and not predominantly bound to the hexadecane. Figures 7(b) and 8(b) show that a significant percentage of the C-atoms are $\mathrm{sp}^{2}$ hybridized indicating the transformation of a part of the lubricant molecules into an amorphous carbon mixed layer.

The state of the lubricated/naked a-C:H layer shown in Fig. 7(b) has a non-sp $\mathrm{s}^{3}$ to $\mathrm{sp}^{3}$ ratio of 5:4 while the lubricated/passivated a-C:H layer shown in Fig. 8(b) has a non-sp $\mathrm{p}^{3}$ to $\mathrm{sp}^{3}$ ratio of 1:1. Figures 7 (c) and $8(\mathrm{c})$ show the intermixing of carbon atoms originally belonging to the hexadecane lubricant and the top and bottom counter bodies. Clearly all carbon sources contribute $C$ atoms to the mixed layer where carbon rings are formed. Figures $7(d)-7(i)$ and $8(d)-8(i)$ show examples of different size carbon rings within the mixed layers shown in Figs. 7(c) and 8(c), respectively. Recalling that all lubricant carbon atoms started as linear molecular chains, Figures 7(d)-7(i) and 8(d)-8(i) demonstrate that a significant portion of the lubricant hexadecane chains are destroyed and rebound to other carbon atoms.

\subsection{Lubricant evolution}

Figure 9 displays the evolution of the hexadecane lubricant molecules during compression and shearing of the ta-C counter bodies with and without $\mathrm{H}$ passivation. Figure 9(a) shows that as sliding progresses the number of $\mathrm{sp}^{3}$ coordinated hexadecane $\mathrm{C}$ atoms decreases while the number of non-sp $\mathrm{p}^{3}$ coordinated lubricant $C$ atoms increases. All hexadecane $C$ atoms are initially $\mathrm{sp}^{3}$ coordinated, therefore the increase in the number of hexadecane $\mathrm{C}$ atoms in a non-sp $\mathrm{p}^{3}$ state indicates that a significant portion of the lubricant molecules degrade as sliding progresses.

The hexadecane $\mathrm{C}$ atoms become $\mathrm{sp}^{2}$ or $\mathrm{sp}^{1}$ coordinated by breaking one $\mathrm{C}-\mathrm{C}$ or one $\mathrm{C}-\mathrm{H}$ bond, or by losing more than one bond and rebonding with other $\mathrm{C}$ or $\mathrm{H}$ atoms within the a-C:H layer. This certainly changes the starting number of $\mathrm{C}-\mathrm{H}$ bonds of every $\mathrm{C}$ atom within the $\mathrm{C}_{16} \mathrm{H}_{34}$ molecules (see Fig. 9(b)). Figure 9(b) shows that there is a noticeable decrease in the number of hexadecane carbon atoms with two $\mathrm{C}-\mathrm{H}$ bonds for both lubricated cases. There is also a noticeable increase in the number of hexadecane $\mathrm{C}$ atoms with only one $\mathrm{C}-\mathrm{H}$ bond while the number of lubricant $\mathrm{C}$ atoms with $3 \mathrm{C}-\mathrm{H}$ bonds (e.g., terminal $\mathrm{C}$ atoms of the hexadecane molecules) remains virtually constant. The hexadecane molecules are degraded sometimes by breaking $\mathrm{C}-\mathrm{C}$ bonds and sometimes by breaking $\mathrm{C}-\mathrm{H}$ bonds as the ta- $\mathrm{C}$ asperities compress and shear the hexadecane lubricant. $\mathrm{C}-\mathrm{H}$ bond breaking can be directly seen in the lubricated/naked case, where all hydrogen stems initially from $\mathrm{CH}_{2}$ or $\mathrm{CH}_{3}$ units of the lubricant, but $\mathrm{C}-\mathrm{H}$ units still form. A few of the dangling $\mathrm{C}$ bonds (that were created by $\mathrm{C}$ or $\mathrm{H}$ abstraction) somewhere in the middle of the $\mathrm{C}_{16} \mathrm{H}_{34}$ molecule can be terminated by dangling $\mathrm{C}$ bonds from other hexadecane molecules or from the evolving a-C:H. This in turn decreases the number of hexadecane $\mathrm{C}$ atoms with two $\mathrm{C}-\mathrm{H}$ bonds and increases the number of hexadecane $C$ atoms with only one C-H bond.

Lastly, Figure 9(c) shows the transformation of a large percentage of the lubricant carbon atoms into carbon rings during the progression of pressing

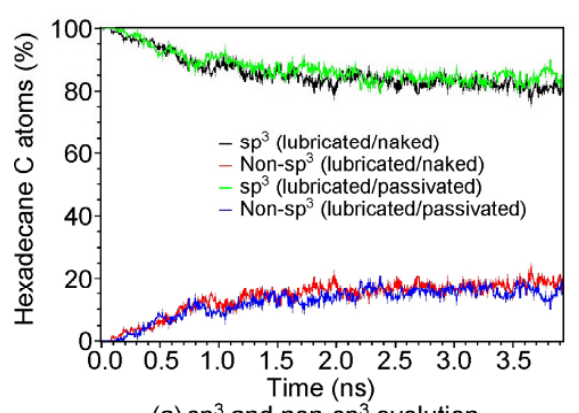

(a) $\mathrm{sp}^{3}$ and non- $\mathrm{sp}^{3}$ evolution

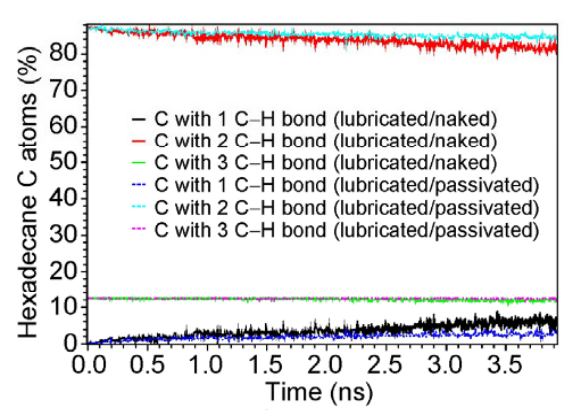

(b) $\mathrm{C}-\mathrm{H}$ bonding

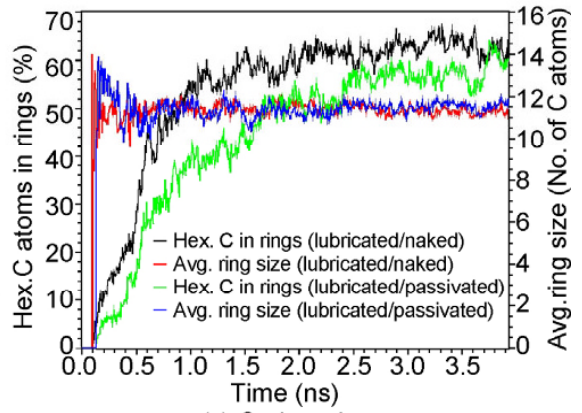

(c) Carbon ring

Fig. 9 Evolution of the hexadecane lubricant carbon atoms during compression and shearing between ta-C counter asperities. (a) Percentage of $\mathrm{sp}^{3}$ and non-sp ${ }^{3}$ hybridized hexadecane $\mathrm{C}$ atoms, (b) degree of $\mathrm{C}-\mathrm{H}$ bonding within the lubricant, and (c) percentage of lubricant $\mathrm{C}$ atoms within rings and average ring size of $\mathrm{C}$ lubricant atoms. 
and sliding. Within about 1 ns about one half of the lubricant carbon atoms in the naked case have been changed from being part of a hexadecane molecule to being part of carbon rings. In the passivated case, half of the hexadecane $\mathrm{C}$ atoms become part of rings after about 2 ns. After about 4 ns, about 60 percent of the hexadecane $\mathrm{C}$ atoms reside in $\mathrm{C}$-ring structures in both cases. The average ring size of rings with hexadecane carbon atoms is about 12 and does not change with time. As shown above in Figs. 7(d)-7(i) and $8(\mathrm{~d})-(\mathrm{i})$, most rings involving hexadecane carbon atoms also contain carbon atoms from the upper and/or lower ta-C counter asperities indicating intimate chemical mixing between the lubricant and surfaces.

\section{Summary and conclusion}

We have carried out classical atomistic simulations of collisions of unpassivated (naked) and H-passivated ta-C asperities in dry and lubricated (hexadecane) conditions. Hydrogen passivation and hexadecane lubrication prevents the counter bodies from cold welding during initial contact under a pressure of 10 GPa. During sliding and flattening of the asperities, surface hydrogen atoms and boundary layers of hexadecane molecules are unable to covalently separate ta-C surfaces and prevent $\mathrm{C}-\mathrm{C}$ bonding during the progression of sliding. As previously reported in Refs. $[8,11,41]$, ta-C counter bodies undergo $\mathrm{sp}^{3}$ to non-sp $\mathrm{p}^{3}$ rehybridization at the sliding interface and eventually form an $\mathrm{sp}^{2}$ rich layer.

The presence of hydrogen or hydrocarbon lubricants reduces the degree of rehybridization. H-passivation and hexadecane molecules decompose and intermix within the $\mathrm{sp}^{2}$ rich layer at the sliding interface. This eventually leads to the formation of an a-C:H material layer that accommodates the sliding motion and significantly reduces the degree of ta-C rehybridization resulting in a thinner tribo-layer during the progression of sliding. The transformation of hexadecane and ta-C surfaces into an a-C:H can be directly observed by monitoring the rehybridization (from $\mathrm{sp}^{3}$ to $\mathrm{sp}^{2}$ ) of the hexadecane carbons, and by the conversion of the linear carbon backbone of the hexadecane to ring structures.

Our simulations show that even though lubricant molecules may decompose, they still have an important function in localizing shear in a thin layer at the surface of the coating. The a-C:H tribomaterial that forms from those decomposed lubricant molecules has a yield strength significantly lower than that of the bulk ta-C. Similar to a molecular lubricant film, this localizes shear and thereby prevents extended subsurface damage to the sample. Softening of the near surface region and shear localization is therefore an important property of amorphous carbon films and may be responsible for their superior wear resistance [8].

Our simulations additionally indicate that instead of coating tribo partners with a-C:H using an expensive plasma-enhanced chemical vapor deposition (PECVD) process such films could in principle be grown in-situ during sliding by polymerizing hydrocarbon lubricants. Unfortunately, our simulations predict that contact pressures of several tens of GPa would be needed for the initiation of the cross linking of the lubricant molecules-pressures that exceed the yield stress of most technical substrates. On the other hand, recent experiments report the catalyzed growth of a-C:H layers from base oil lubricants during sliding [44]. Most likely, in the experiment catalyst particles facilitate the bond breaking within the lubricant thus reducing the normal pressures necessary for the polymerization reaction.

\section{Acknowledgements}

This work was supported by the BMWi with the Progect Pegasus II (M. M.) and by the European Commission (Marie-Curie IOF 272619 for L. P.). Simulations were carried out at the Jülich Supercomputing Centre (JSC). Analysis and visualization was partially carried out with Ovito [45].

Open Access: This article is distributed under the terms of the Creative Commons Attribution License which permits any use, distribution, and reproduction in any medium, provided the original author(s) and source are credited.

\section{References}

[1] Robertson J. Diamond-like amorphous carbon. Mater Sci Eng $R$ 37: 129-281 (2002) 
[2] Erdemir A, Donnet C. Tribology of diamond-like carbon films: Recent progress and future prospects. $J$ Phys $D$ 39: R311-R327 (2006)

[3] Donnet C, Erdemir A. Tribology of Diamond-Like Carbon Films: Fundamentals and Applications. Springer US, 2008.

[4] Cho S, Chasiotis I, Friedmann T A, Sullivan J P. Young's modulus, Poisson's ratio and failure properties of tetrahedral amorphous diamond-like carbon for MEMS devices. $J$ Micromech Microeng 15: 728-735 (2005)

[5] Konicek A R, Grierson D S, Gilbert P U P A, Sawyer W G, Sumant A V, Carpick R W. Origin of ultra-low friction and wear in ultrananocrystalline diamond. Phys Rev Lett 100: 235502 (2008)

[6] Konicek A R, Grierson D S, Sumant A V, Friedmann T A, Sullivan J P, Gilbert P U P A, Sawyer W G, Carpick R W. Influence of surface passivation on the friction and wear behavior of ultrananocrystalline diamond and tetrahedral amorphous carbon thin films. Phys Rev B 85: 155448 (2012)

[7] Joly-Pottuz L, Matta C, de Barros Bouchet M I, Vacher B, Martin J M, Sagawa T. Superlow friction of taC lubricated by glycerol: An electron energy loss spectroscopy study. J Appl Phys 102: 064912 (2007)

[8] Kunze T, Posselt M, Gemming S, Seifert G, Konicek A, Carpick R, Pastewka L, Moseler M. Wear, plasticity, and rehybridization in tetrahedral amorphous carbon. Tribol Lett 53: 119-126 (2014)

[9] Matta C, De Barros Bouchet M I, Le-Mogne T, Vachet B, Martin J M, Sagawa T. Tribochemistry of tetrahedral hydrogen-free amorphous carbon coatings in the presence of OH-containing lubricants. Lubr Sci 20: 137-149 (2008)

[10] Harrison J A, Brenner D W. Simulated tribochemistry-An atomic-scale view of the wear of diamond. J Am Chem Soc 116: 10399-10402 (1994)

[11] Gao G T, Mikulski P T, Harrison J A. Molecular-scale tribology of amorphous carbon coatings: Effects of film thickness, adhesion, and long-range interactions. $\mathrm{J} \mathrm{Am} \mathrm{Chem}$ Soc 124: 7202-7209 (2002)

[12] Pastewka L, Moser S, Moseler M. Atomistic insights into the running-in, lubrication, and failure of hydrogenated diamondlike carbon coatings. Tribol Lett 39: 49-61 (2010)

[13] Schall J D, Gao G, Harrison J A. Effects of adhesion and transfer film formation on the tribology of self-mated DLC contacts. J Phys Chem C 114: 5321-5330 (2010)

[14] Hyun S, Pei L, Molinari J-F, Robbins M O. Finite-element analysis of contact between elastic self-affine surfaces. Phys Rev E 70: 026117 (2004)

[15] Persson B N J, Albohr O, Tartaglino U, Volokitin A I, Tosatti E. On the nature of surface roughness with application to contact mechanics, sealing, rubber friction and adhesion. J Phys: Condens Matter 17: R1-R26 (2005)
[16] Carbone G, Bottiglione F. Asperity contact theories: Do they predict linearity between contact area and load? J Mech Phys Solids 56: 2555-2572 (2008).

[17] Pastewka L, Robbins M O. Contact between rough surfaces and a criterion for macroscopic adhesion. Proc Natl Acad Sci USA 111: 3298-3303 (2014)

[18] Moras G, Pastewka L, Walter M, Schnagl J, Gumbsch P, Moseler M. Progressive shortening of sp-hybridized carbon chains through oxygen-induced cleavage. J Phys Chem C 115: 24653-24661 (2011)

[19] Moras G, Pastewka L, Gumbsch P, Moseler M. Formation and oxidation of linear carbon chains and their role in the wear of carbon materials. Tribol Lett 44: 355-365 (2011)

[20] M'ndange-Pfupfu A, Eryilmaz O, Erdemir A, Marks L. Quantification of sliding-induced phase transformation in $\mathrm{N}_{3} \mathrm{FC}$ diamond-like carbon films. Diam Relat Mater 20: 1143-1148 (2011)

[21] Mndange-Pfupfu A, Ciston J, Eryilmaz O, Erdemir A, Marks L. Direct observation of tribochemically assisted wear on diamond-like carbon thin films. Tribol Lett 49: 351-356 (2013)

[22] Zilibotti G, Righi M C, Ferrario M. Ab initio study on the surface chemistry and nanotribological properties of passivated diamond. Phys Rev B 79: 075420 (2009)

[23] Zilibotti G, Corni S, Righi M C. Load-induced confinement activates diamond lubrication by water. Phys Rev Lett 111: 146101 (2013)

[24] Brenner D W, Shenderova O A, Harrison J A, Stuart S J, Ni B, Sinnott S B. A secondgeneration reactive empirical bond order (REBO) potential energy expression for hydrocarbons. J Phys: Condens Matter 14: 783-802 (2002)

[25] Pastewka L, Pou P, Pérez R, Gumbsch P, Moseler M. Describing bond-breaking processes by reactive potentials: The importance of an environment-dependent interaction range. Phys Rev B 78: 161402(R) (2008)

[26] Pastewka L, Mrovec M, Moseler M, Gumbsch P. Bond order potentials for fracture, wear and plasticity. MRS Bulletin 37: 493-503 (2012)

[27] Pastewka L, Klemenz A, Gumbsch P, Moseler M. Screened empirical bond-order potentials for Si-C. Phys Rev B 87: 205410 (2013)

[28] Nayak P R. Random process model of rough surfaces. $J$ Tribol 93: 398-407 (1971)

[29] Greenwood J A. A unified theory of surface roughness. Proc R Soc Lond A 393: 133-157 (1984)

[30] Bitzek E, Koskinen P, Gähler F, Moseler M, Gumbsch P. Structural relaxation made simple. Phys Rev Lett 97: 170201 (2006)

[31] Allen M P, Tildesley D J. Computer Simulation of Liquids. New York: Oxford University Press, 1989. 
[32] Stuart S J, Tutein A B, Harrison J A. A reactive potential for hydrocarbons with intermolecular interactions. $J$ Chem Phys 112: 6472-6486 (2000)

[33] Soddemann T, Dunweg B, Kremer K. Dissipative particle dynamics: A useful thermostat for equilibrium and nonequilibrium molecular dynamics simulations. Phys Rev E 68: 46702 (2003)

[34] Hoogerbrugge P J, Koelman J M V A. Simulating microscopic hydrodynamic phenomena with dissipative particle dynamics. Europhys Lett 19: 155-160 (1992)

[35] Ferrari A C, Robertson J. Interpretation of Raman spectra of disordered and amorphous carbon. Phys Rev B 61: 14095 (2000)

[36] Franzblau D S. Computation of ring statistics for network models of solids. Phys Rev B 44: 4925-4930 (1991).

[37] Fu X-Y, Falk M L, Rigney D A. Sliding behavior of metallic glass-Part II. Computer simulations. Wear 250: 420-430 (2001)

[38] Fu X-Y, Rigney D A, Falk M L. Sliding and deformation of metallic glass: experiments and MD simulations. J NonCryst Solids 317: 206-214 (2003)

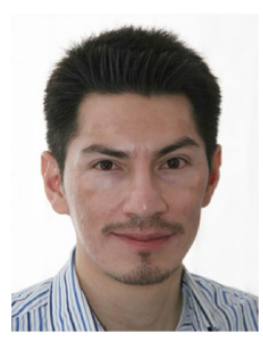

Pedro A. ROMERO. He has received a BS degree in Mechanical Engineering (2001), a MS degree in Solids Mechanics (2004), and a PhD in Mechanical Engineering (2008) all from Rutgers University (New Brunswick, NJ, USA) where he performed solid mechanics research using continuum

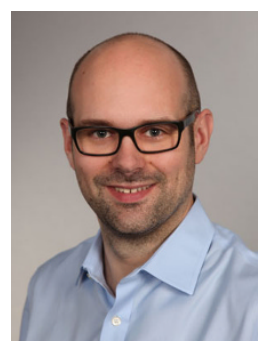

Lars PASTEWKA. He has received an MS degree in physics (2003), a Dipl.-Ing. in Microsystems Engineering (2005) and a $\mathrm{PhD}$ degree in physics (2010), the former from North Carolina State University and the latter ones from the University of Freiburg. Pastewka has worked at
[39] Pastewka L, Moser S, Moseler M, Blug B, Meier S, T Hollstein, P Gumbsch. The running-in of amorphous hydrocarbon tribocoatings: A comparison between experiment and molecular dynamics simulations. Int $J$ Mat Res 99: 1136-1143 (2008).

[40] Pastewka L, Peguiron J, Gumbsch P, Moseler M. Molecular dynamics simulation of gold solid film lubrication. Int $J$ Mat Res 101: 981-988 (2010)

[41] Pastewka L, Moser S, Gumbsch P, Moseler M. Anisotropic mechanical amorphization drives wear in diamond. Nature Mater 10: 34-38 (2011)

[42] Falk M L. Molecular-dynamics study of ductile and brittle fracture in model noncrystalline solids. Phys Rev B 60: 7062-7070 (1999)

[43] Falk M L, Langer J S. From simulation to theory in the physics of deformation and fracture. MRS Bull 20: 40-45 (2000)

[44] Erdemir, A. Private communication.

[45] Stukowski A. Visualization and analysis of atomistic simulation data with OVITO-The open visualization tool. Modelling Simul Mater Sci Eng 18: 015012 (2010)

level approaches, i.e, FEM and micromechanical modeling. After a two-year post-doctoral stay at École Polytechnique Fédérale de Lausanne (EPFL) (Lausanne, Switzerland) where he worked on atomistic modeling of nanomaching, he has spent the last three years at the Fraunhofer Institut für Werkstoffmechanik (IWM) (Freiburg, Germany) investigating tribological problems using atomistic simulations.

Fraunhofer IWM and has held Fulbright and MarieCurie fellowships that allowed him to carry out research at North Carolina State and Johns Hopkins University. He recently moved to the Institute for Applied Materials at the Karlsruhe Institute of Technology to study contact, friction and wear using a range of theoretical methods from the atomic to the continuum scale. 


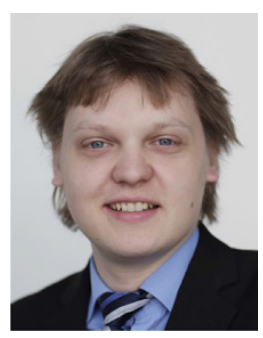

Julian VON LAUTZ. He holds a Dipl.-Ing. in Mechanical Engineering (2010) from the Technical University of Braunschweig. He is currently a $\mathrm{PhD}$ candidate at Karlsruhe Institute

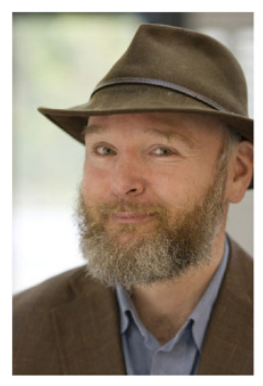

Michael MOSELER. He received his diploma and $\mathrm{PhD}$ degrees in Physics from the Albert-LudwigsUniversity in Freiburg, Germany in 1994 and 1998, respectively. After his PostDoc at the Georgia Institute of Technology in Atlanta, he became leader of a computational materials of Technology and a graduate researcher at the Fraunhofer Institute for Mechanics of Materials (IWM) in Freiburg, Germany where he investigates the tribological properties of amorphous carbon and diamond.

science group at the Fraunhofer Institute for Mechnanics of Materials in Freiburg in 2002. In addition to this position he is Professor for Modelling and Simulation of Functional Nanosystems at the Physics Department of the Albert-Ludwigs-University in Freiburg. His research areas cover atomistic simulations in tribology, nano technology and cluster science. 